\title{
Identifying Prognostic Lncrnas Based on A ceRNA Regulatory Network in Laryngeal Squamous Cell Carcinoma
}

Yong Shi ( $\sim$ biosy1993@163.com )

Zhengzhou University

Dongli Yang

Shanxi Medical University

Yixiao Qin

Shanxi Medical University

\section{Research Article}

Keywords: LncRNA, CeRNA, Prognosis, Laryngeal squamous cell carcinoma

Posted Date: January 25th, 2021

DOI: https://doi.org/10.21203/rs.3.rs-147155/v1

License: (c) This work is licensed under a Creative Commons Attribution 4.0 International License.

Read Full License 


\section{Abstract}

Purpose Growing evidence demonstrates that long non-coding RNAs (IncRNAs) play a crucial role as competing endogenous RNAs (ceRNAs) in tumor occurrence. The IncRNAs' function and clinical significance in laryngeal squamous cell carcinoma (LSCC) remain unclear. The study aims to reveal the IncRNA-associated ceRNA regulatory network in LSCC and clarify its clinical relevance.

Methods Here, we obtained LSCC transcriptome sequencing data from The Cancer Genome Atlas (TCGA) database and identified the differential expression profile of IncRNAs, miRNAs, and mRNAs by the EdgeR $R$ package. The function enrichment analysis of mRNAs was performed using clusterProfiler $R$ package and GSEA3.0. Then, we constructed a ceRNA network and a prognosis model based on IncRNAs through bioinformatic methods. Moreover, we explored the functions of prognosis-related IncRNA in LSCC by CCK8 and transwell assay.

Results 1961 IncRNAs, 69 miRNAs, and 2224 mRNAs were identified as differentially expressed genes in LSCC tissues. According to the transcriptome differential expression profile, a ceRNA network containing 61 IncRNAs, 21 miRNAs, and 77 mRNAs was established. Then, four IncRNAs (AC011933.2, FAM30A, LINC02086, LINC02575) were extracted from the ceRNA network to build a prognosis model for LSCC patients. Furthermore, we found that LINC02086 and LINC02575 promoted the proliferation, migration, and invasion of LSCC cells while AC011933.2 and FAM30A inhibited these biological functions in vitro.

Conclusion Four IncRNAs from the ceRNA network were abnormally expressed and related to patient prognosis in LSCC. They played a significant role in the progress of LSCC via affecting the proliferation and metastasis of tumor cells.

\section{Introduction}

Laryngeal squamous cell carcinoma (LSCC) is a typical head and neck squamous cell carcinoma [1]. The conventional treatment for LSCC, including chemotherapy, radiation therapy, and surgical resection, have a satisfied curative effect on early-stage patients[2]. However, patients with advanced LSCC continuously have a low overall five-year survival rate[3]. Therefore, it is necessary to reveal the pathogenesis of LSCC and improve the level of diagnosis and treatment.

Non-coding RNAs (ncRNAs) are a type of RNA that cannot encode proteins, including small non-coding RNA (sncRNAs) and long non-coding RNA (IncRNAs) [4, 5]. MicroRNAs (miRNAs) that belong to sncRNAs and have been widely studied decreases the expression level of targeted mRNAs by binding to its 3 'untranslated regions (3'-UTRs) to degrade mRNAs [6, 7]. Long non-coding RNAs (IncRNAs), the length of over 200 nucleotides (nt), can play significant regulation functions in several biological processes, including transcription, pretranscription, chromatin modification, translation, post-translation[8-10]. Currently, researchers found that the abnormal expression of IncRNAs is involved in the occurrence and development of malignant tumors[11]. For example, IncRNA HOTAIR is upregulated in many cancer and promotes the proliferation, migration, and invasion of tumor cells, including breast cancer, rectal cancer, 
pancreatic cancer, and kidney cancer[12-15]. Upregulated- LINC02410 and served as a diagnostic marker in rectal cancer [16].

The ceRNA hypothesis, a hot topic of IncRNA research, is that some IncRNAs sharing the same miRNA response elements (MREs) with mRNAs bind miRNAs competitively to block the interaction between miRNA and mRNA, thereby the expression level of mRNAs has been elevated $[17,18]$. It has been reported that the ceRNA regulatory patterns extensively present in many types of cancers[19]. For example, the IncRNA FAM225A upregulates ITGB3 by adsorbing miR-590-3p/miR-1275 to promote NPC cells' proliferation and invasion [20]. IncRNA PVT1 acts as a ceRNA to adsorb miR-143 and upregulates the expression of HK2 to encourage the proliferation and metastasis of GBC cells[21]. However, the IncRNAassociated ceRNA network in LSCC remains unclear. So, this study aims to build a ceRNA regulatory network for a better understanding of IncRNA's molecular mechanism in LSCC.

In this study, the LSCC transcriptome sequencing data was extracted from the TCGA database, including the RNA expression profile of 111 tumor samples and 12 normal samples. Using bioinformatics tools, we constructed a ceRNA network containing 61 IncRNAs, 21 miRNAs, and 77 mRNAs. Meanwhile, a fourIncRNA prognosis model based on the ceRNA network was also established. Furthermore, we found that the four IncRNAs had apparent influences on the proliferation, migration, and invasion of LSCC cells in vitro. The above analysis and experiment results show that the four IncRNAs may be served as prognosis biomarkers and become Therapeutic targets of LSCC in the future.

\section{Materials And Methods}

\section{Samples and patients from TCGA}

Transcriptome sequencing data of patients with laryngeal squamous cell carcinoma (LSCC) was obtained from the TCGA database (https://portal.gdc.cancer.gov/), which contained 111 LSCC samples and 12 normal samples. Besides, the corresponding clinical characteristics of LSCC patients' overall survival (OS) were obtained from the TCGA database.

\section{Identification of differentially expressed IncRNA, mRNA, and miRNA}

Ensemble IDs of the genes were transformed into gene symbols based on GENCODE (https://www.gencodegenes.org/human/). Differentially expressed IncRNAs (DElncRNAs), differentially expressed mRNAs (DEmRNAs), and differentially expressed miRNA (DEmiRNAs) between LSCC samples and normal samples were identified by the EdgeR $R$ package[22]. We regarded IncRNAs, mRNAs, and miRNAs as DElncRNAs, DEmRNAs, and DEmiRNAs when they met these criteria $(p<0.05$, and $\| \log 2 \mathrm{FC} \mid>2)$.

\section{Functional enrichment analysis}

Through clusterProfiler R package[23], We performed Gene Ontology (G0) and Kyoto Encyclopedia of Genes and Genomes (KEGG) analysis. GSEA analysis was performed by GSEA3.0 
(http://software.broadinstitute.org/gsea/index.jsp), and the cut-off criteria for gene sets were set to adjust $p<0.05$.

\section{Construction of IncRNA-miRNA-mRNA ceRNA network}

DEIncRNAs, DEmiRNAs, and DEmRNAs were used to establish the ceRNA network. Firstly, we screened the DEIncRNA-DEmiRNA pairs using Starbase[24]. Secondly, TargetScan[25], miRDB[26], and miRwalk[27] were used to screen the DEmiRNA-DEmRNA pairs. When DEmiRNA-DEmRNA pairs were predicted in all three databases, we considered candidates for constructing the ceRNA network. Ultimately, Cytoscape 3.6.1 was used to build a IncRNA-related ceRNA network[28].

\section{Screening of LSCC prognostic signatures}

Survival analysis and univariate cox regression analysis were performed to explore the correlation between the IncRNAs from the ceRNA network and OS of LSCC patients by the survival R package[29]. A prognosis model was built by multivariate regression analysis. And the following formula was used to calculate the prognostic risk score of LSCC patients: Risk score = 3 IncRNA1 * explncRNA1 + $\beta$ IncRNA2 * explncRNA2 $+\ldots+\beta$ IncRNAn * explncRNAn (' $\beta$ ' is the regression coefficient of IncRNAs and 'exp' is the expression of corresponding IncRNAs). Through the survivalROC R package, a receiver operating characteristic (ROC) curve was drawn to assess the prognosis model's accuracy.

\section{Cell culture and transfection}

Human laryngeal cancer cell lines TU177, Hep-2 were purchased from Bena Culture Collection (Beijing, China). All cells were cultured in Dulbecco's Modified Eagle Medium (DMEM; Gibco, USA) with 10\% Fetal Bovine Serum (FBS; Gibco, USA) at $37^{\circ} \mathrm{C}$ and $5 \% \mathrm{CO}_{2}$. The cells were transfected with prognosis relatedIncRNA using Lipofectamine ${ }^{\mathrm{TM}} 3000$ (Thermo Fisher Scientific, USA).

\section{Quantitative real-time PCR}

According to the manufacturer's protocol, the total RNA was extracted from cells by TRIzol reagent (Invitrogen, CA, USA). qRT-PCR analysis on IncRNA was performed using HiScript ${ }^{\circledR}$ II Q Select RT SuperMix for qPCR Kit (vazyme, Nanjing, China) and ChamQ Universal SYBR qPCR Master Mix (vazyme, Nanjing, China). $18 \mathrm{~S}$ was used as the endogenous control.

\section{CCK-8 assay}

Cell proliferation was examined by Cell Counting Kit-8 (CCK-8; GLPBIO, USA). The cells were incubated in the 96 -well plates $\left(1 \times 10^{3}\right.$ cells per well) for 24 hours, and then $10 \mu$ l CCK- 8 reagents were added to each well. Cell viability was determined by detecting the absorbance at $450 \mathrm{~nm}$.

\section{Cell migration and invasion assay}


Transwell chambers with 8.0um pore polycarbonate membrane insert (Corning, USA) assessed cell migration and invasion abilities. For cell invasion assay, $40 \mu \mathrm{l}$ matrigel solution (Matrigel: medium=1:4) was added to transwell inserts and solidified for $3 \mathrm{~h}$ at $37^{\circ} \mathrm{C}$. Then $500 \mu \mathrm{L}$ DMEM with $10 \%$ FBS was added to the lower chamber. Cells were resuspended with serum-free medium and plated into transwell inserts at

$1 \times 10^{5} \mathrm{cells}$ / well. The cells on the filter's upper surface were removed after they were cultured at $37^{\circ} \mathrm{C}$ for 24-72h. For cell migration assay, the matrigel solution was not needed on the inserts, and other steps were as same as invasion assay. Cells on the lower surface of the filter were fixed with $4 \%$ paraformaldehyde for 20 mins and then washed twice with PBS before they were stained with $1 \%$ crystal violet solution for $10 \mathrm{mins}$. The stained cells were counted by a microscope.

\section{Statistical analysis}

the statistical analysis software included R 3.4.3 and SPASS 22.0. All measurement data were presented as mean \pm SD. Two-tailed Student's t-test was used to compare two groups. P-value $<0.05$ was considered statistically significant.

\section{Results}

\section{Identification of DElncRNAs, DEmiRNAs, and DEmRNAs}

Considering the cut points of |log2-fold change| $>2$ and $p<0.05,1961$ DElncRNAs, 69 DEmiRNAs, and 2224 DEmRNAs were identified to be differentially expressed genes in LSCC tissues. Among them, 1398 IncRNAs, 32 miRNAs, and 1285 mRNAs were the high expression genes, while 563 IncRNAs, 37 miRNAs, and 939 mRNAs were the low expression genes in LSCC (Supplementary Material 1). The differences in the expression of IncRNAs, miRNAs, and mRNAs in LSCC were demonstrated on volcano plots (Fig. 1A, 1B, 1C). The top 20 DElncRNAs, DEmiRNAs, DEmRNAs expression profiles in heatmaps, respectively (Fig. 1D, 1E, 1F).

\section{DEmRNAs functional enrichment analysis}

For exploring the function and molecular mechanism of DEmRNAs in the occurrence and development of laryngeal squamous cell carcinoma, we performed GO and KEGG enrichment analysis on DEmRNAs (Supplementary Material 2). The top $20 \mathrm{KEGG}$ pathways and top $20 \mathrm{GO}$ biological process terms $(p<0.05)$, sorted by the $p$-values, were chosen for Bar graph and bubble graph (Fig. 2A, 2B). Among these pathways and biological process terms, some terms containing ECM-receptor interactions, Tight junction, and PPAR signaling pathway are correlated to the proliferation and metastasis of tumors. In addition, KEGG-GSEA analysis showed that DEmRNAs gene sets enrich in Human papillomavirus infection, $\mathrm{PI} 3 \mathrm{~K}$ Akt signaling pathway, Alcoholism, Neuroactive ligand-receptor interaction, Cytokine-cytokine receptor interaction (Fig. 2C, 2E; adjust $p<0.05$; Supplementary Material 3). GO biological process GSEA analysis results had no statistical significance (Fig. 2D; adjust $p>0.05$ ). 
To explore the ceRNA Molecular regulatory network centered on IncRNA in LSCC, we constructed the ceRNA network according to the workflow (Fig. 3A). First, the interactions between IncRNAs and miRNAs were predicted by the starbase database among the DElncRNAs and DEmiRNAs. We obtained 168 IncRNA-miRNA pairs (Supplementary Material 4). Next, the interactions between miRNAs and mRNA were screened by TargetScan, miRDB, and miRWalk among the DEmiRNAs and DEmRNAs. We obtained 118 miRNA-mRNA pairs that all interactions exist in three databases (Supplementary Material 4). Then we used Cytoscape software to construct a IncRNA-miRNA-mRNA ceRNA network consisting of 61 IncRNAs, 21 miRNAs, and 77 mRNAs with a total of 179 interactions, based on the IncRNA-miRNA pairs and the miRNA-mRNA pairs (Fig. 3B).

\section{Screening IncRNA-associated prognosis factors based on ceRNA network}

Kaplan-Meier analysis results showed that six IncRNAs, including AC011933.2, LINC00689, LINC02570, FAM225B, LINC02086, and LINC02575 were closely associated with overall survival (OS) of patients (Fig. $4 ; p<0.05)$. furthermore, Univariate cox regression analysis and multivariate cox regression analysis based on DEIncRNAs from ceRNA network were performed to construct a prognosis model as follows: risk score $=$ relative expression of $A C 011933.2 *(-0.36287)+$ relative expression of $\operatorname{FAM}_{30} A^{*}(-0.12015)+$ relative expression of LINC02086*(0.12687) + relative expression of LINC02575*(0.11148). (Fig. 5) was a forest plot for the four IncRNAs consisted of the prognosis model. The survival risk score of all LSCC patients was presented in (Fig. 6A, 6C). (Fig. 6B) was a survival state graph for all LSCC patients. For detecting the specificity and sensitivity of the model. The Kaplan-Meier analysis result showed that the high-risk group's survival rate tended to lower than the low-risk group (Fig. 7A; $p<0.05$ ). The ROC analysis results showed that the area under curve (AUC) value was 0.609 in the 1 st year, 0.793 in the 3rd year, 0.752 in the 5 th year, 0.876 in the 7th year, 0.915 in the 9th year, which indicated that the cox risk model has high specificity and sensitivity (Fig .7B-7F).

\section{Prognosis-related IncRNAs affected the proliferation, migration, and invasion of LSCC cells}

To understand the role of prognosis-related IncRNAs in the oncogenesis of LSCC, we elevated the expression level of prognosis-related IncRNAs by transfected pcDNA3.1(+)-prognosis-related IncRNAs into LSCC cells. The results showed that all four IncRNAs expression increased in the experimental groups compared to the control groups (Fig. 8A, 8B). The CCK-8 assay demonstrated that both LINC02086 and LINC02575 promoted Hep-2 and TU 177 proliferation while both AC011933.2 and FAM30A inhibited Hep2 and TU 177 proliferation (Fig. 8C, 8D; $p<0.05$ ). Then, the migration and invasion were detected by transwell assay. The results revealed that both LINC02086 and LINC02575 promoted Hep-2 and TU 177 cell migration and invasion while both AC011933.2 and FAM30A inhibited Hep-2 and TU 177 migration and invasion (Fig. 8E, 8F; p <0.05).

\section{Discussion}

LSCC, a type of common head and neck squamous cell carcinomas, causes a severe threat to peoples' health all over the world[30]. As the incidence of laryngeal squamous cell carcinoma is increasing year by 
year, it is urgent to clarify the pathogenesis and identify significant prognosis biomarkers for improving the current treatment to LSCC. Our study demonstrated that a four-IncRNA prognosis model based on the ceRNA network had a sufficient ability to predict the prognosis of LSCC. Moreover, the four IncRNAs played a significant role in the proliferation and metastasis of LSCC. Therefore, these prognosis-related IncRNAs may be served as a new therapeutic target in the future.

LncRNA plays a vital role in the occurrence and development of tumors. Recently, more and more studies have shown that the ceRNA molecular regulation model is a common way for IncRNA to promote or inhibit tumor growth [31]. For example, ABHD11-AS1 serves as a competitive endogenous RNA to upregulate STAT3 by sponging miR-1301-3p in PTC[32]. Linc-DYNC2H1-4 promotes EMT and CSC phenotypes by acting as a sponge of miR-145 in pancreatic cancer cells[33]. However, most previous studies are limited to a single molecular regulation axis, and there is a lack of comprehensive and indepth research on molecular regulation networks. As we know, the interaction between IncRNA and miRNA is not the only correspondence relationship. One IncRNA may have many different MREs, and other IncRNAs may also have the same MREs. For example, Inc HOTAIR can act as a molecular sponge for both miR-206 and miR-124[15, 34]. Both IncRNA TUG1 and IncRNA PVT1 can interact with miR$145[35,36]$. Similarly, the interaction between miRNA and mRNA is not the only correspondence relationship. Therefore, it is helpful for a comprehensive understanding of the molecular mechanism of IncRNA to elucidate the regulatory network of IncRNA-miRNA-mRNA.

Our study determined 1961 IncRNAs, 69 miRNAs, and 2224 mRNAs, which were abnormal expression genes in LSCC according to the transcriptome sequencing data derived from the TCGA. Then, functional enrichment analysis, including GO, KEGG, and GSEA, were performed by bioinformatics tools. We found that DEmRNAs were enriched in some tumor-related biological processes or pathways such as ECMreceptor interactions, Tight junction, PPAR signaling pathway, Human papillomavirus infection, PI3K-Akt signaling pathway. Next, a IncRNA-miRNA-mRNA ceRNA network was constructed according to DElncRNAs, DEmRNAs, and DEmiRNAs, and it included 61 IncRNAs, 21 miRNAs, and 77 mRNAs. Furthermore, we extracted a four-IncRNA (AC011933.2, FAM30A, LINC02086, LINC02575) prognosis model from the ceRNA network. These results indicate that the ceRNA network is useful for uncovering the molecular mechanism of IncRNA in LSCC and may become prognosis biomarkers of LSCC.

Several risk score systems were established to predict LSCC patients' prognosis in previous studies [37, 38]. However, all these prognosis biomarkers based on the predicting model have not been verified by experiments in vitro. Our study constructed a four-IncRNA prognosis model and confirmed the biological functions of the four IncRNAs in LSCC.

The four IncRNAs' functions in the progress of LSCC remain unclear because of the few associated studies. In our research, we found that the expression of AC011933.2 and FAM30A is abnormal in LSCC, and they have a negative correlation with the OS of LSCC patients. On the contrary, LINC02086 and LINC02575 are positively correlated with the overall survival of LSCC patients. Furthermore, the four IncRNAs were found to promote or inhibit LSCC cell proliferation, migration, and invasion in vitro. 
Therefore, the four IncRNA may be considered as new tumor suppressor genes and oncogenes that deserve further research.

Inevitably, our research has several innate limitations that need to be addressed. Although the bioinformatics process of constructing the ceRNA network was designed reasonably, and experiments were performed in vitro to verify the functions of prognosis-associated IncRNAs, our study's main disadvantage was the lack of validation of the IncRNA-miRNA-mRNA molecular regulatory axis extracted from the ceRNA network. Besides, our cox risk model was based on data derived from the TCGA database, and clinical validation of larger samples is necessary. Despite the above drawbacks, our study results show that our cox risk model with 4 IncRNAs play a significant role in the occurrence and development of LSCC.

\section{Conclusion}

In conclusion, we have identified a IncRNA-related prognosis model that can effectively predict OS in LSCC. Moreover, we verified these IncRNAs could influence the biological function of LSCC cells.

\section{Declarations}

\section{ACKNOWLEDGMENT}

This study was supported by the Shanxi Province Graduate Education Innovation Project (program no. 2018SY042).

\section{FUNDING}

Shanxi Province Graduate Education Innovation Project, Grant/Award Number: 2018 SY042.

\section{COMPETING INTERESTS}

The authors declare that there is no conflict of interest.

\section{AVAILABILITY OF DATA AND MATERIALS}

All data generated or analysed during this study are obtained from TCGA database (https://portal.gdc.cancer.gov/).

\section{ETHICS APPROVAL AND CONSENT TO PARTICIPATE}

Not applicable.

\section{CONSENT FOR PUBLICATION}

Not applicable. 


\section{AUTHOR CONTRIBUTIONS}

YS designed this research plan. DY collected and analyzed data. YQ completed the experiment in vitro. YS wrote the manuscript. All authors read and approved the final manuscript.

\section{References}

1. Siegel RL, Miller KD, Jemal A (2019) Cancer statistics, 2019. CA Cancer J Clin 69 (1):7-34. doi:10.3322/caac. 21551

2. Baird BJ, Sung CK, Beadle BM, Divi V (2018) Treatment of early-stage laryngeal cancer: A comparison of treatment options. Oral Oncol 87. doi:10.1016/j.oraloncology.2018.09.012

3. Miller KD, Nogueira L, Mariotto AB, Rowland JH, Yabroff KR, Alfano CM, Jemal A, Kramer JL, Siegel RL (2019) Cancer treatment and survivorship statistics, 2019. CA Cancer J Clin 69 (5):363-385. doi:10.3322/caac. 21565

4. Romano G, Veneziano D, Acunzo M, Croce CM (2017) Small non-coding RNA and cancer. Carcinogenesis 38 (5):485-491. doi:10.1093/carcin/bgx026

5. Slack FJ, Chinnaiyan AM (2019) The Role of Non-coding RNAs in Oncology. Cell 179 (5):1033-1055. doi:10.1016/j.cell.2019.10.017

6. Lu TX, Rothenberg ME (2018) MicroRNA. J Allergy Clin Immunol 141 (4):1202-1207. doi:10.1016/j.jaci.2017.08.034

7. Dong H, Lei J, Ding L, Wen Y, Ju H, Zhang X (2013) MicroRNA: function, detection, and bioanalysis. Chem Rev 113 (8):6207-6233. doi:10.1021/cr300362f

8. Ulitsky I, Bartel DP (2013) lincRNAs: genomics, evolution, and mechanisms. Cell 154 (1):26-46. doi:10.1016/j.cell.2013.06.020

9. Kopp F, Mendell JT (2018) Functional Classification and Experimental Dissection of Long Noncoding RNAs. Cell 172 (3):393-407. doi:10.1016/j.cell.2018.01.011

10. St Laurent G, Wahlestedt C, Kapranov P (2015) The Landscape of long non-coding RNA classification. Trends Genet 31 (5):239-251. doi:10.1016/j.tig.2015.03.007

11. Bhan A, Soleimani M, Mandal SS (2017) Long Noncoding RNA and Cancer: A New Paradigm. Cancer Res 77 (15):3965-3981. doi:10.1158/0008-5472.CAN-16-2634

12. Gupta RA, Shah N, Wang KC, Kim J, Horlings HM, Wong DJ, Tsai M-C, Hung T, Argani P, Rinn JL, Wang Y, Brzoska P, Kong B, Li R, West RB, van de Vijver MJ, Sukumar S, Chang HY (2010) Long noncoding RNA HOTAIR reprograms chromatin state to promote cancer metastasis. Nature 464 (7291):1071-1076. doi:10.1038/nature08975

13. Kim K, Jutooru I, Chadalapaka G, Johnson G, Frank J, Burghardt R, Kim S, Safe S (2013) HOTAIR is a negative prognostic factor and exhibits pro-oncogenic activity in pancreatic cancer. Oncogene 32 (13):1616-1625. doi:10.1038/onc.2012.193 
14. Kogo R, Shimamura T, Mimori K, Kawahara K, Imoto S, Sudo T, Tanaka F, Shibata K, Suzuki A, Komune S, Miyano S, Mori M (2011) Long non-coding RNA HOTAIR regulates polycomb-dependent chromatin modification and is associated with poor prognosis in colorectal cancers. Cancer Res 71 (20):6320-6326. doi:10.1158/0008-5472.CAN-11-1021

15. Pan Y, Wu Y, Hu J, Shan Y, Ma J, Ma H, Qi X, Jia L (2018) Long non-coding RNA HOTAIR promotes renal cell carcinoma malignancy through alpha-2, 8-sialyltransferase 4 by sponging microRNA-124. Cell Prolif 51 (6):e12507. doi:10.1111/cpr.12507

16. Zhao Y, Du T, Du L, Li P, Li J, Duan W, Wang Y, Wang C (2019) Long non-coding RNA LINC02418 regulates MELK expression by acting as a ceRNA and may serve as a diagnostic marker for colorectal cancer. Zhang et al. Hum Genomics 10 (8):568. doi:10.1038/s41419-019-1804-x

17. Tay Y, Rinn J, Pandolfi PP (2014) The multilayered complexity of ceRNA crosstalk and competition. Nature 505 (7483):344-352. doi:10.1038/nature12986

18. Sanchez-Mejias A, Tay Y (2015) Competing endogenous RNA networks: tying the essential knots for cancer biology and therapeutics. J Hematol Oncol 8:30. doi:10.1186/s13045-015-0129-1

19. Qi X, Zhang D-H, Wu N, Xiao J-H, Wang X, Ma W (2015) ceRNA in cancer: possible functions and clinical implications. J Med Genet 52 (10):710-718. doi:10.1136/jmedgenet-2015-103334

20. Zheng Z-Q, Li Z-X, Zhou G-Q, Lin L, Zhang L-L, Lv J-W, Huang X-D, Liu R-Q, Chen F, He X-J, Kou J, Zhang J, Wen X, Li Y-Q, Ma J, Liu N, Sun Y (2019) Long Noncoding RNA FAM225A Promotes Nasopharyngeal Carcinoma Tumorigenesis and Metastasis by Acting as ceRNA to Sponge miR-5903p/miR-1275 and Upregulate ITGB3. Cancer Res 79 (18):4612-4626. doi:10.1158/0008-5472.CAN19-0799

21. Chen J, Yu Y, Li H, Hu Q, Chen X, He Y, Xue C, Ren F, Ren Z, Li J, Liu L, Duan Z, Cui G, Sun R (2019) Long non-coding RNA PVT1 promotes tumor progression by regulating the miR-143/HK2 axis in gallbladder cancer. Mol Cancer 18 (1):33. doi:10.1186/s12943-019-0947-9

22. Robinson MD, McCarthy DJ, Smyth GK (2010) edgeR: a Bioconductor package for differential expression analysis of digital gene expression data. Bioinformatics 26 (1):139-140. doi:10.1093/bioinformatics/btp616

23. Yu G, Wang LG, Han Y, He QY (2012) clusterProfiler: an R package for comparing biological themes among gene clusters. OMICS 16 (5):284-287. doi:10.1089/omi.2011.0118

24. Li J-H, Liu S, Zhou H, Qu L-H, Yang J-H (2014) starBase v2.0: decoding miRNA-ceRNA, miRNA-ncRNA and protein-RNA interaction networks from large-scale CLIP-Seq data. Nucleic Acids Res 42 (Database issue):D92-D97. doi:10.1093/nar/gkt1248

25. Agarwal V, Bell GW, Nam J-W, Bartel DP (2015) Predicting effective microRNA target sites in mammalian mRNAs. Elife 4. doi:10.7554/eLife.05005

26. Chen $Y$, Wang $X(2020)$ miRDB: an online database for prediction of functional microRNA targets. Nucleic Acids Res 48 (D1):D127-D131. doi:10.1093/nar/gkz757

27. Sticht C, De La Torre C, Parveen A, Gretz N (2018) miRWalk: An online resource for prediction of microRNA binding sites. PLoS ONE 13 (10):e0206239. doi:10.1371/journal.pone.0206239 
28. Otasek D, Morris JH, Boucas J, Pico AR, Demchak B (2019) Cytoscape Automation: empowering workflow-based network analysis. Genome Biol 20 (1):185. doi:10.1186/s13059-019-1758-4

29. Rizvi AA, Karaesmen E, Morgan M, Preus L, Wang J, Sovic M, Hahn T, Sucheston-Campbell LE (2019) gwasurvivr: an R package for genome-wide survival analysis. Bioinformatics 35 (11):1968-1970. doi:10.1093/bioinformatics/bty920

30. Cossu AM, Mosca L, Zappavigna S, Misso G, Bocchetti M, De Micco F, Quagliuolo L, Porcelli M, Caraglia M, Boccellino M (2019) Long Non-coding RNAs as Important Biomarkers in Laryngeal Cancer and Other Head and Neck Tumours. Int J Mol Sci 20 (14). doi:10.3390/ijms20143444

31. Schmitz SU, Grote P, Herrmann BG (2016) Mechanisms of long non-coding RNA function in development and disease. Cell Mol Life Sci 73 (13):2491-2509. doi:10.1007/s00018-016-2174-5

32. Wen J, Wang H, Dong T, Gan P, Fang H, Wu S, Li J, Zhang Y, Du R, Zhu Q (2019) STAT3-induced upregulation of IncRNA ABHD11-AS1 promotes tumour progression in papillary thyroid carcinoma by regulating miR-1301-3p/STAT3 axis and PI3K/AKT signalling pathway. Cell Prolif 52 (2):e12569. doi:10.1111/cpr.12569

33. Gao Y, Zhang Z, Li K, Gong L, Yang Q, Huang X, Hong C, Ding M, Yang H (2017) Linc-DYNC2H1-4 promotes EMT and CSC phenotypes by acting as a sponge of miR-145 in pancreatic cancer cells. Cell Death Dis 8 (7):e2924. doi:10.1038/cddis.2017.311

34. Li T, Qin Y, Zhen Z, Shen H, Cong T, Schiferle E, Xiao S (2019) Long non-coding RNA HOTAIR/microRNA-206 sponge regulates STC2 and further influences cell biological functions in head and neck squamous cell carcinoma. Cell Prolif 52 (5):e12651. doi:10.1111/cpr.12651

35. Gu W, Yuan Y, Wang L, Yang H, Li S, Tang Z, Li Q (2019) Long non-coding RNA TUG1 promotes airway remodelling by suppressing the miR-145-5p/DUSP6 axis in cigarette smoke-induced COPD. J Cell Mol Med 23 (11):7200-7209. doi:10.1111/jcmm.14389

36. Shen S-N, Li K, Liu Y, Yang C-L, He C-Y, Wang H-R (2019) Down-regulation of long non-coding RNA PVT1 inhibits esophageal carcinoma cell migration and invasion and promotes cell apoptosis via microRNA-145-mediated inhibition of FSCN1. Mol Oncol 13 (12):2554-2573. doi:10.1002/18780261.12555

37. Kong X, Qi J, Yan Y, Chen L, Zhao Y, Fang Z, Fan J, Liu M, Liu Y (2019) Comprehensive analysis of differentially expressed profiles of IncRNAs, mRNAs, and miRNAs in laryngeal squamous cell carcinoma in order to construct a ceRNA network and identify potential biomarkers. J Cell Biochem 120 (10):17963-17974. doi:10.1002/jcb.29063

38. Liu Y, Ye F (2019) Construction and integrated analysis of crosstalking ceRNAs networks in laryngeal squamous cell carcinoma. PeerJ 7:e7380. doi:10.7717/peerj.7380

\section{Figures}



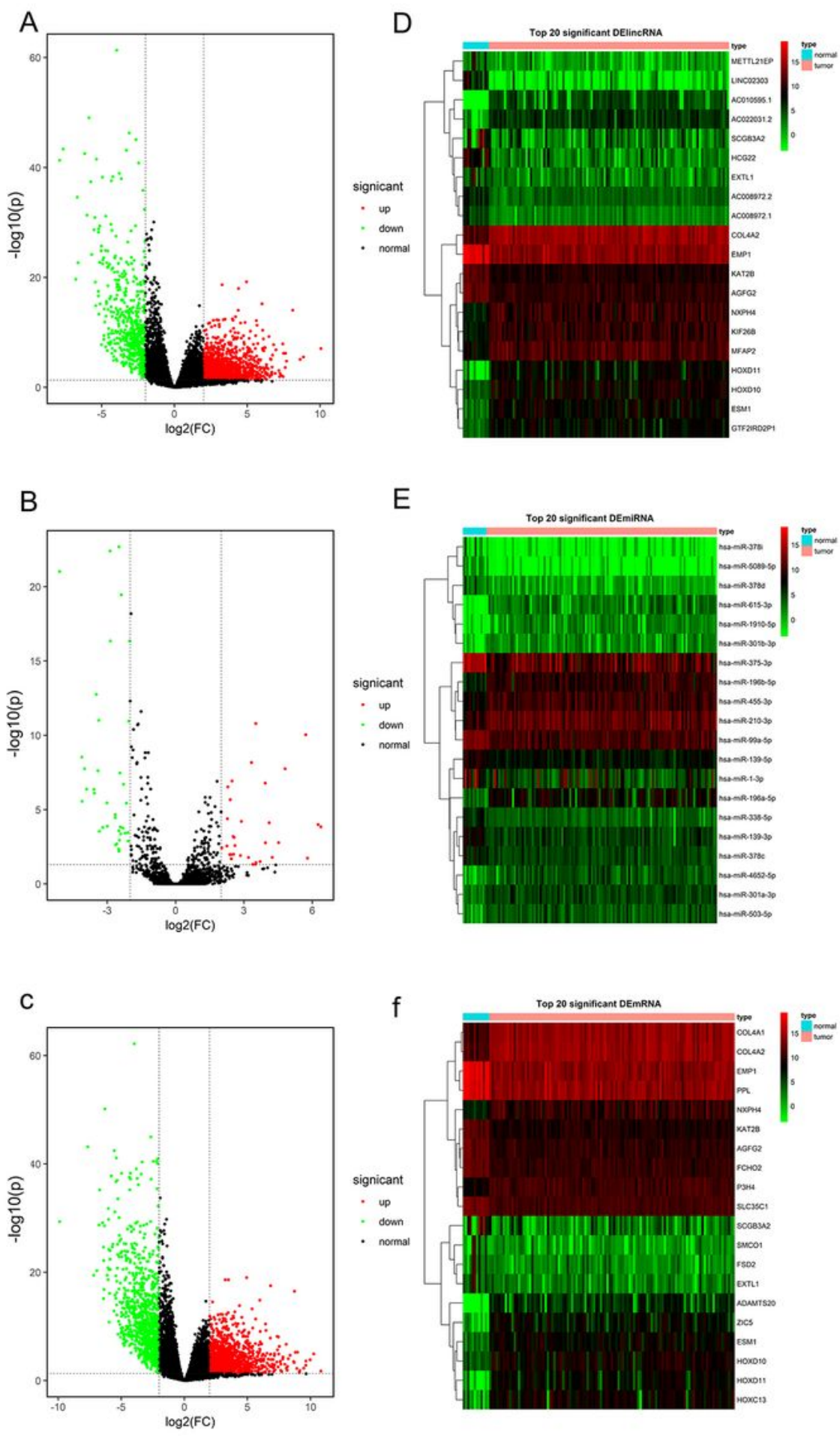

Figure 1

Volcano plots and heatmaps show DElncRNAs, DEmiRNAs, and DEmRNAs. (A-C) Volcano plots for DElncRNAs, DEmiRNAs, and DEmRNAs. (E-F) heatmaps for DElncRNAs, DEmiRNAs, and DEmRNAs. 

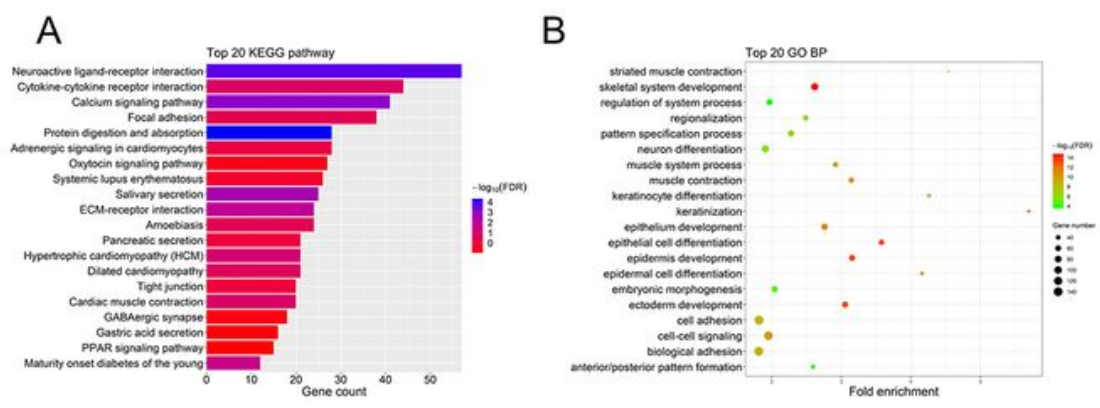

C

D
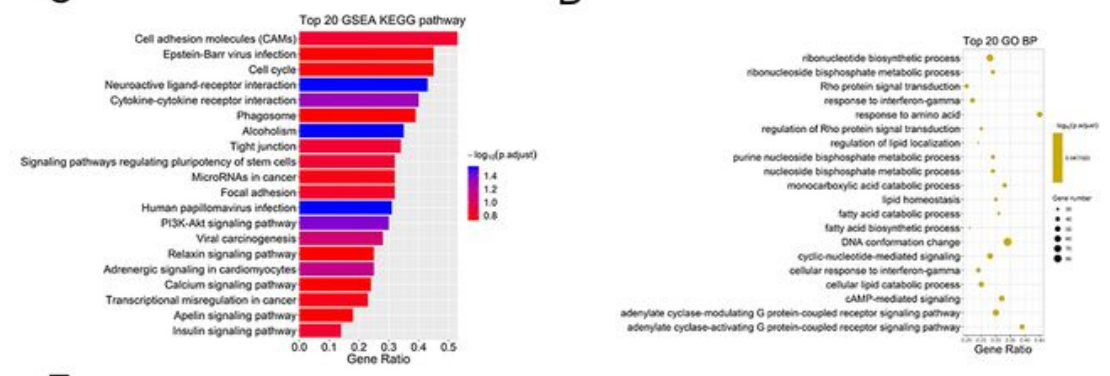

$\mathrm{E}$
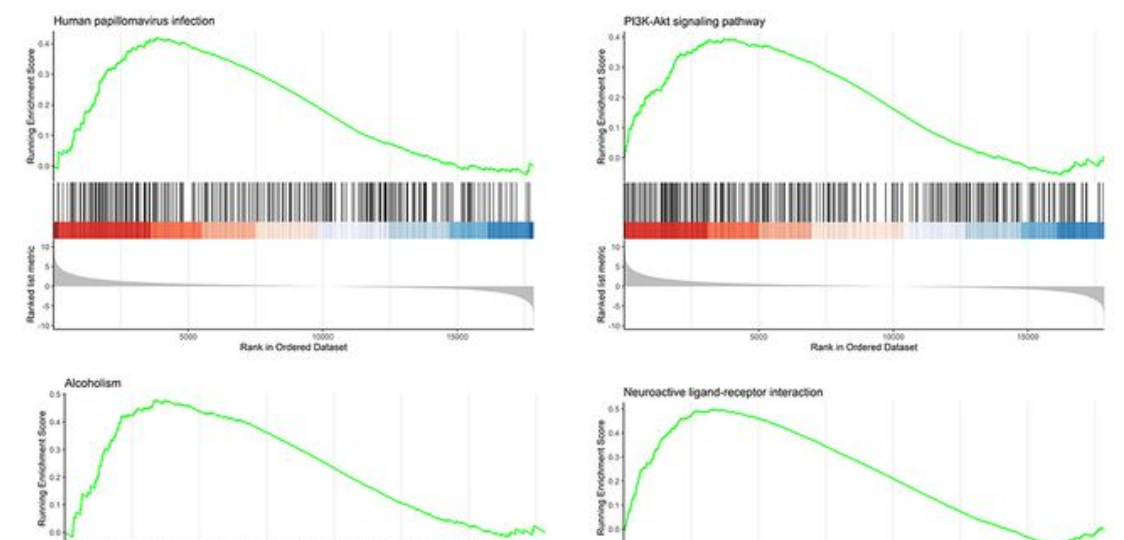

||||||||||||||||||||||||||||||||||||||||||||||||||||||.
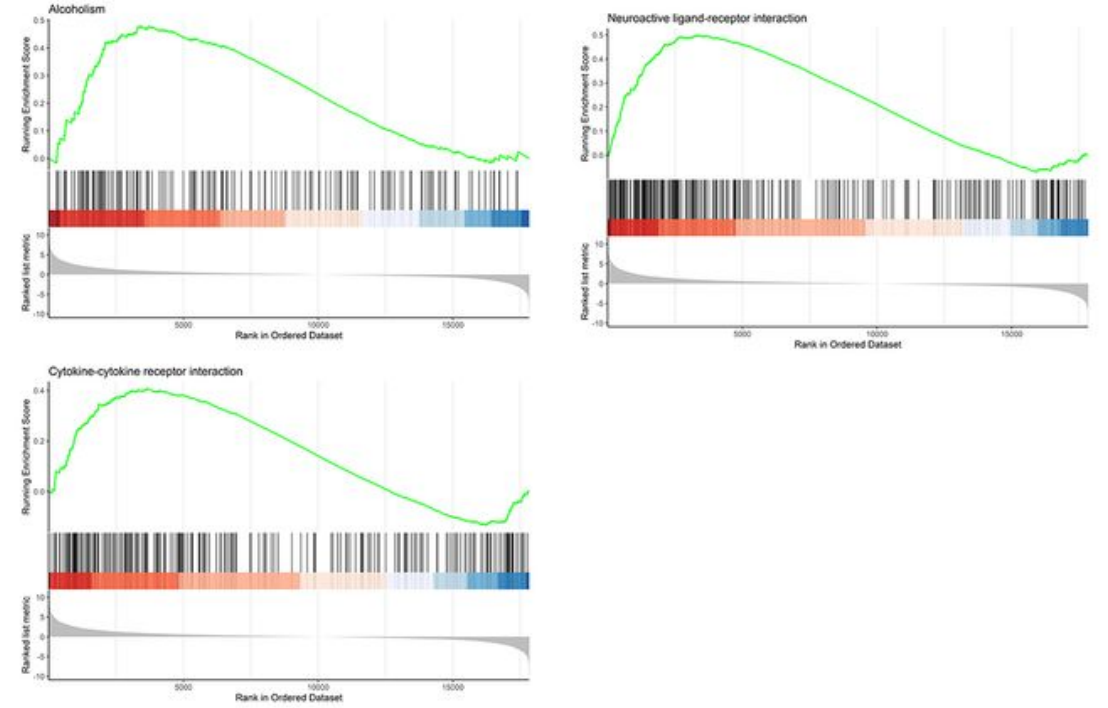

Figure 2

GO and KEGG pathway function enrichment analysis for DEmRNAs. (A) Top 20 KEGG pathways based on the p-value. (B) Top $20 \mathrm{GO}$ BP terms based on p values. (C) Top 20 KEGG-GSEA pathway based on adjust p-values. (D) Top 20 GO BP-GSEA terms based on adjust p-values. (E) Five statistically significant KEGGGSEA pathways with adjust $p<0.05$. 
A
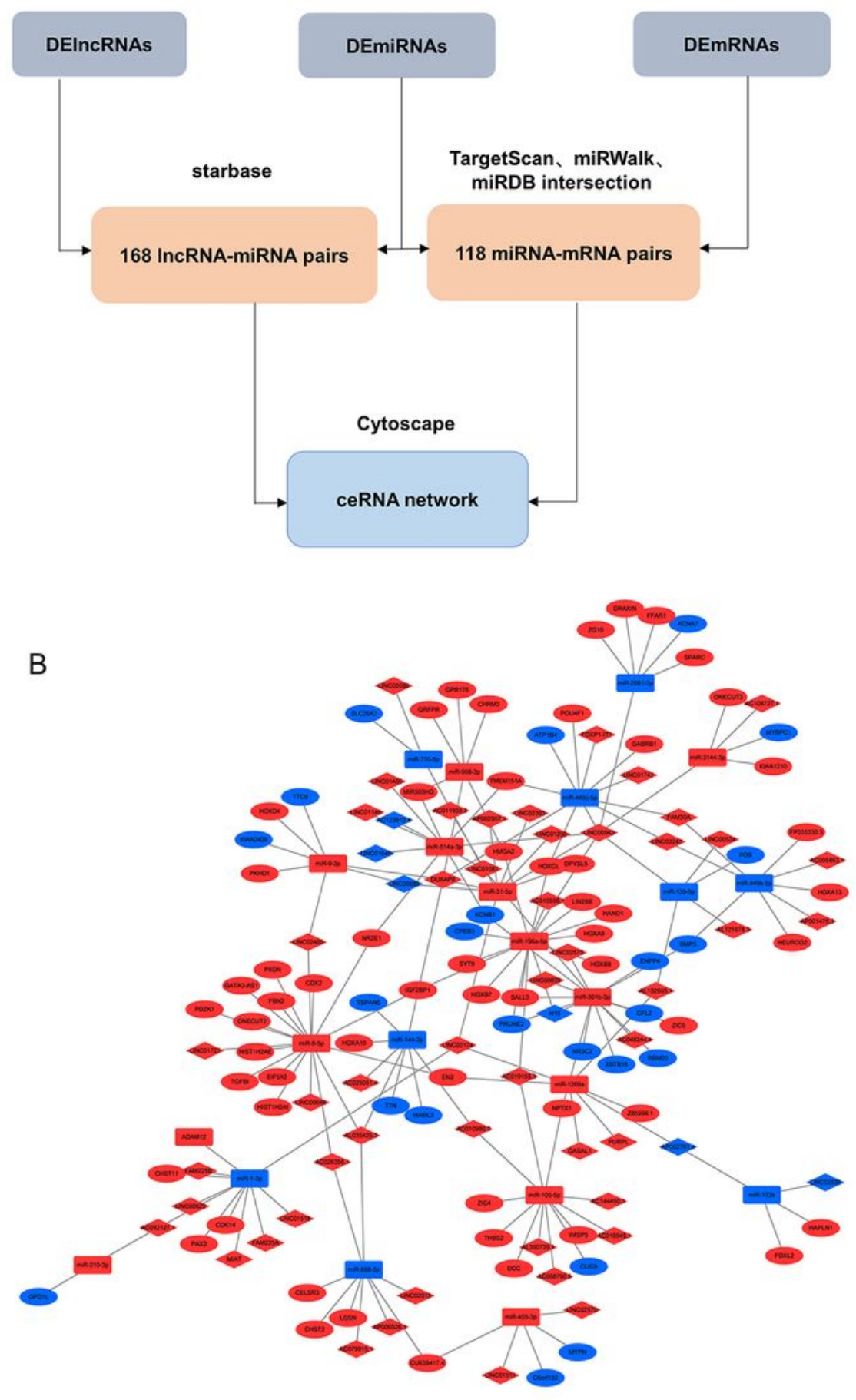

\section{Figure 3}

Construction of ceRNA network in LSCC. (A) The workflow of building ceRNA network. (B) CeRNA network regulation model. The diamond represents IncRNA. Rectangle represents miRNA. Oval represents mRNA. Red represents high expression in cancer tissues. Blue represents low expression in cancer tissues. 
A

Survival curve $(p=6.342262 e-03)$

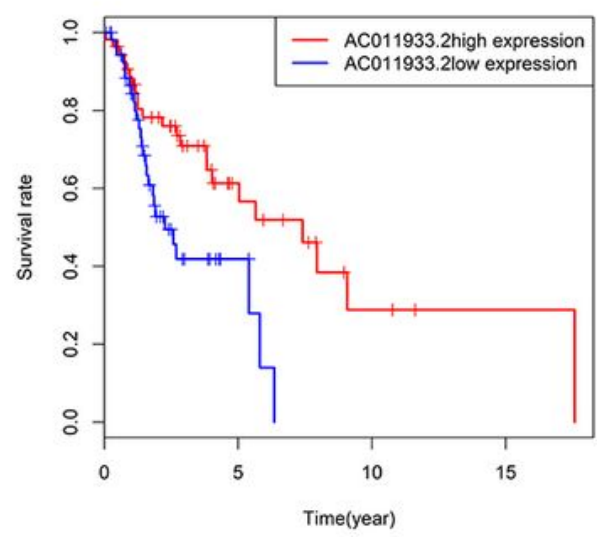

C

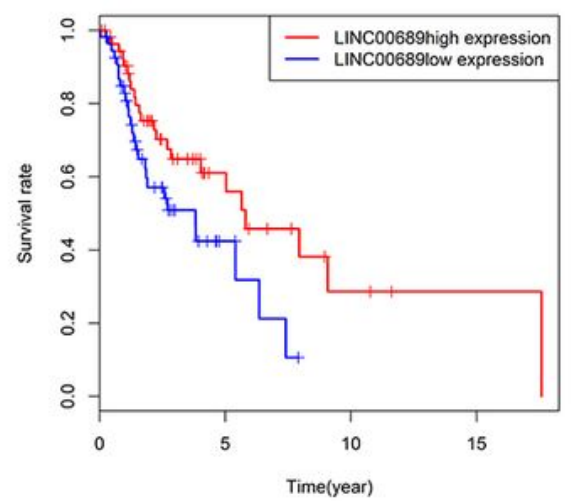

E

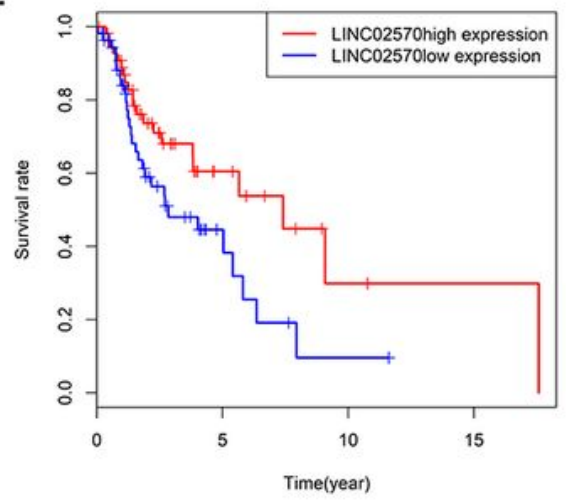

B Survival curve $(p=3.48802 \mathrm{e}-02)$

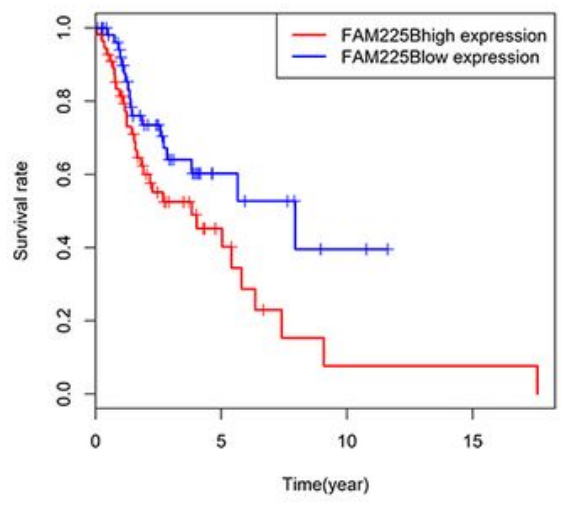

D Survival curve $(p=3.249039 e-02)$
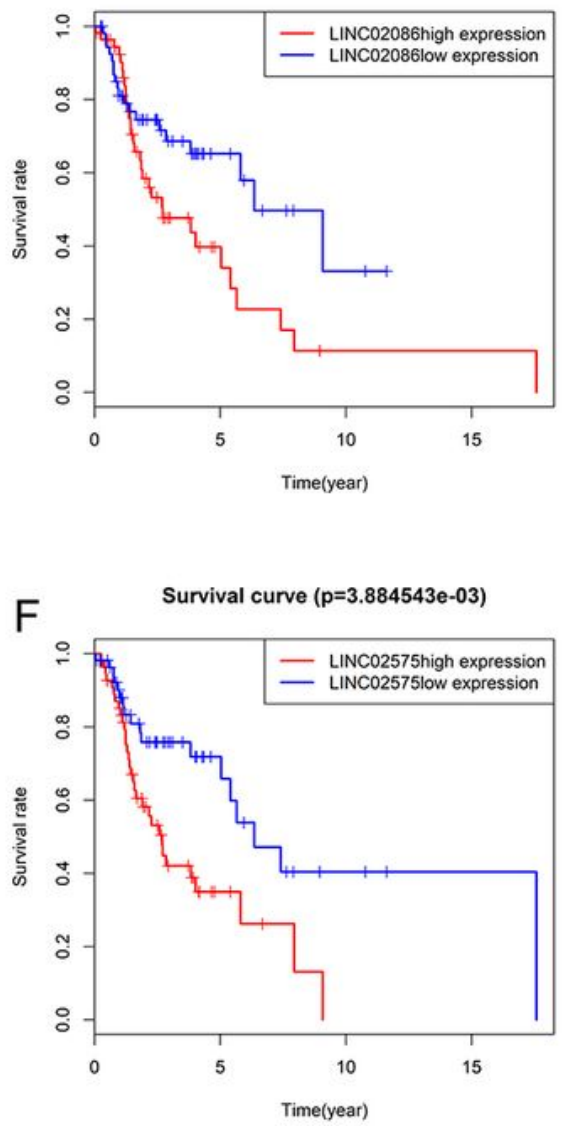

\section{Figure 4}

Kaplan-Meier survival curves of six IncRNAs (log-rank p<0.05). 111 LSCC patients were divided into two groups (high expression and low expression) according to the median of relative expression value. 


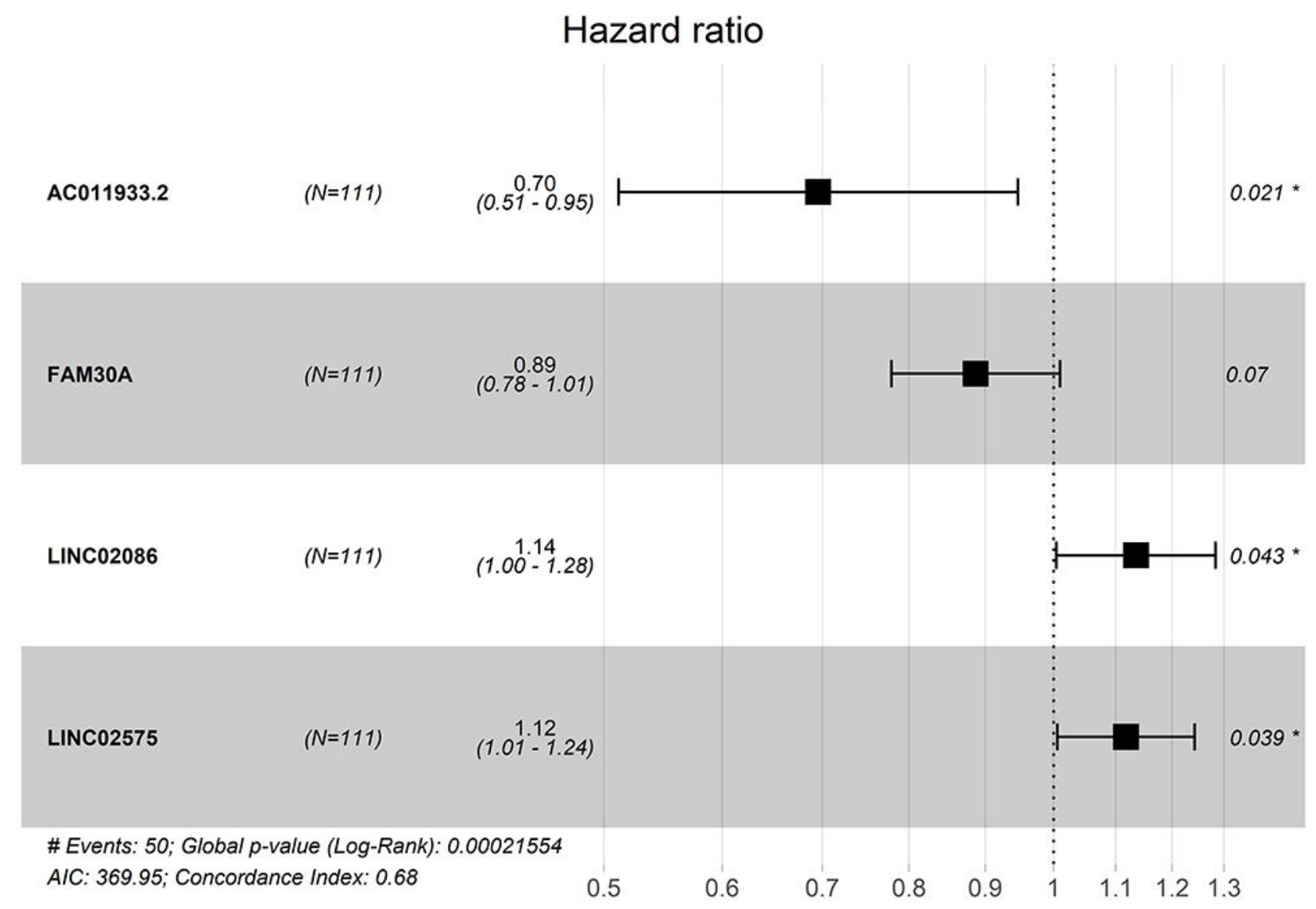

Figure 5

Forecast plots for the four prognosis-related IncRNAs. 
A

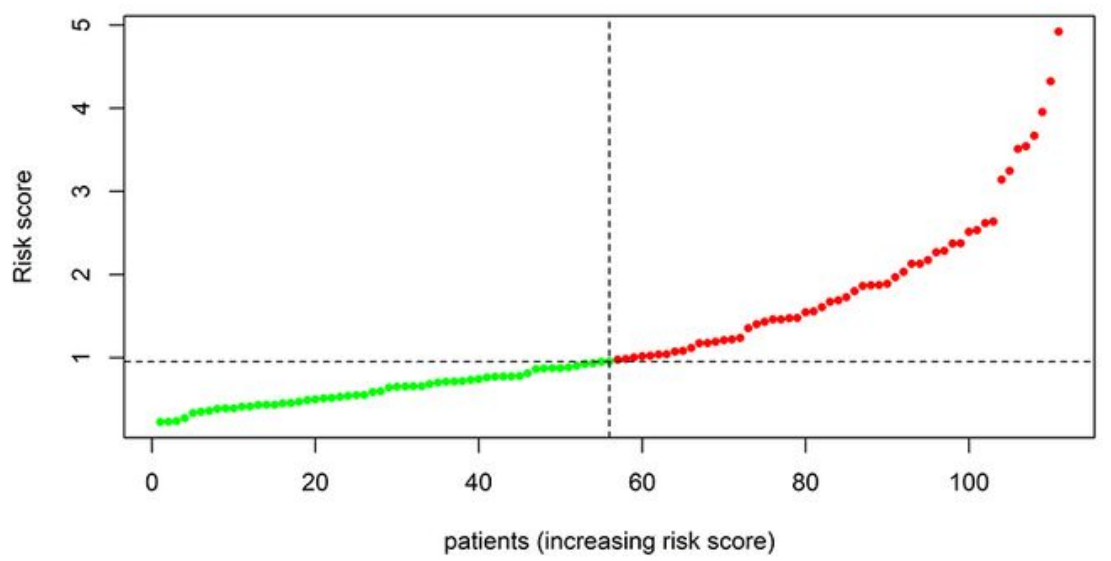

B

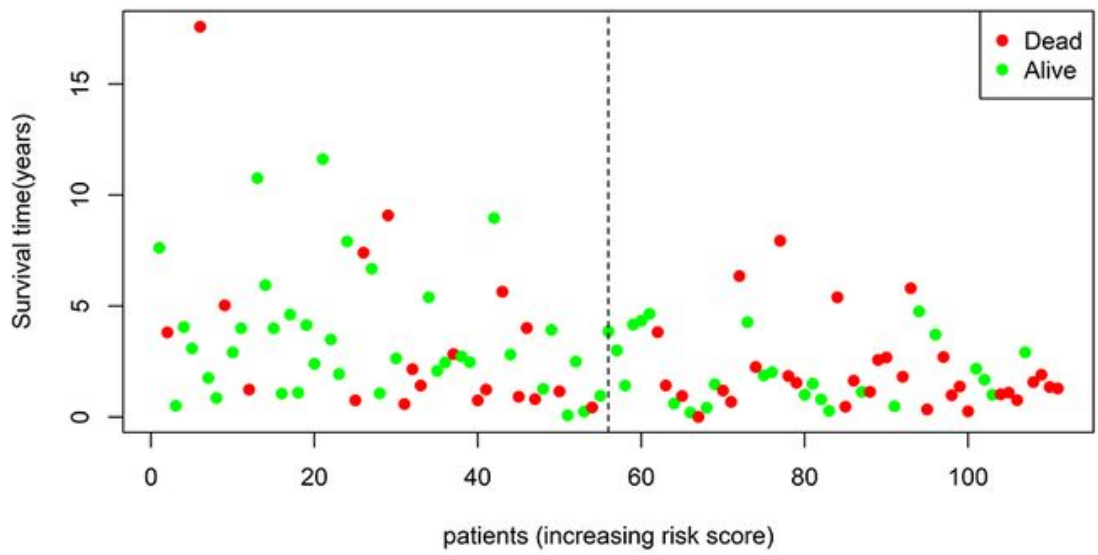

C

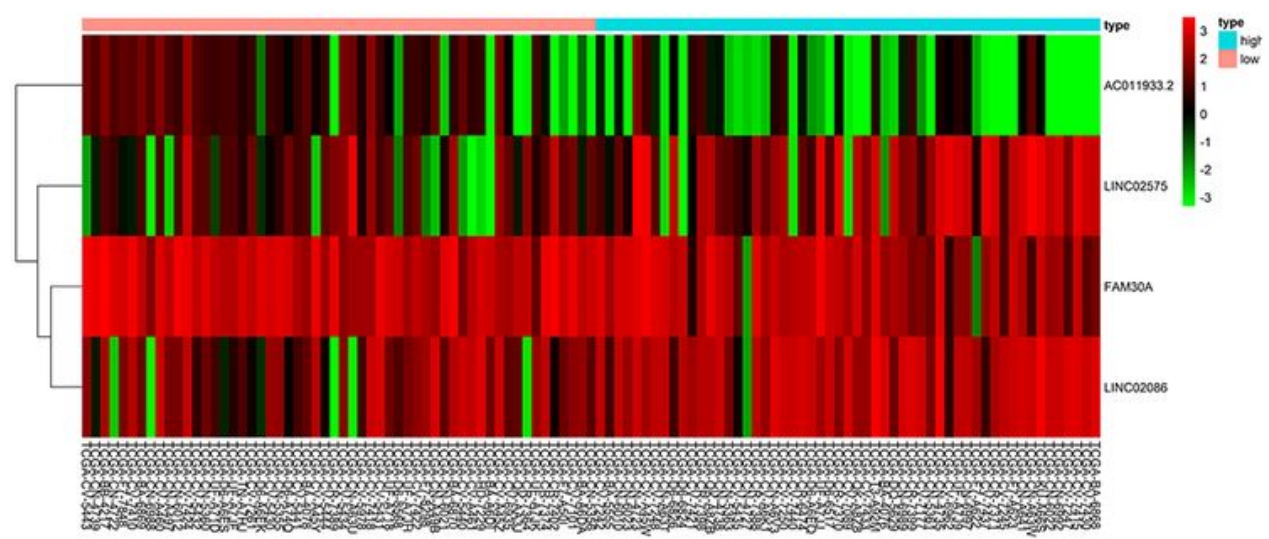

Figure 6

Risk score of all patients with LSCC. (A) Risk score plots of 111 patients. (B) survival state of 111 patients. (C) Risk score heatmap for 111 patients. 
A

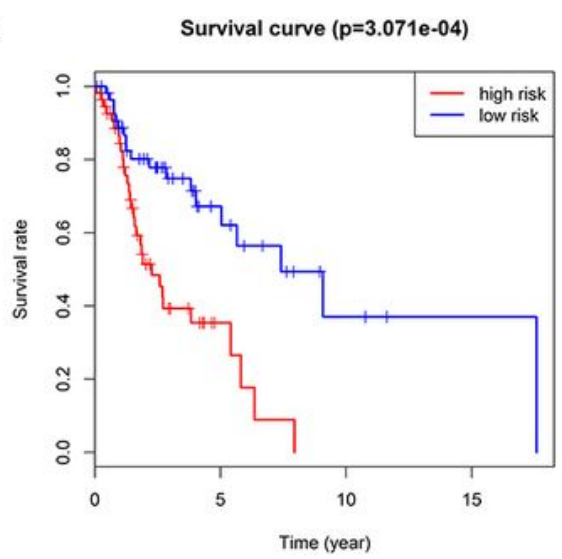

C

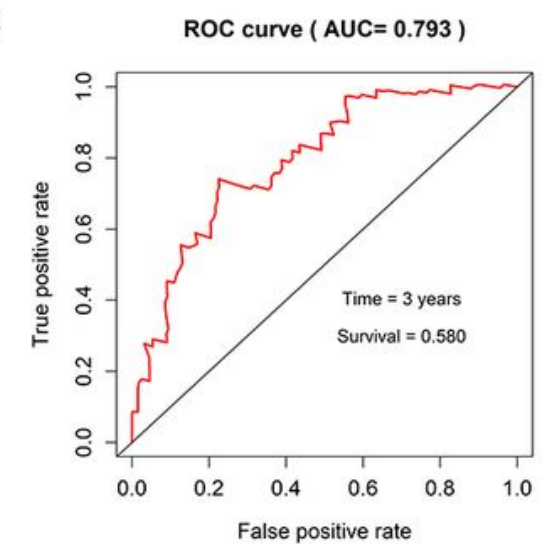

$E$

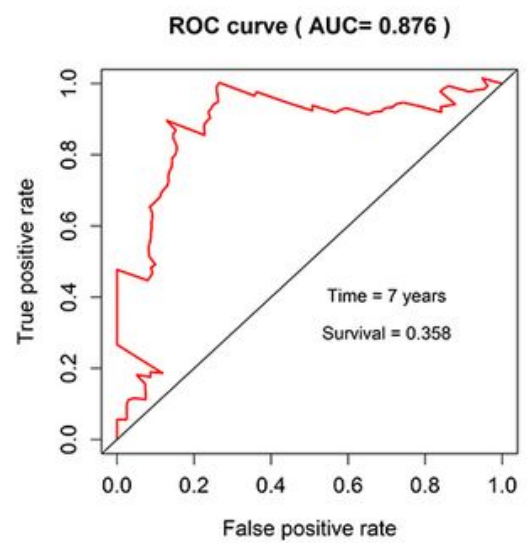

B ROC curve ( $A \cup C=0.609)$

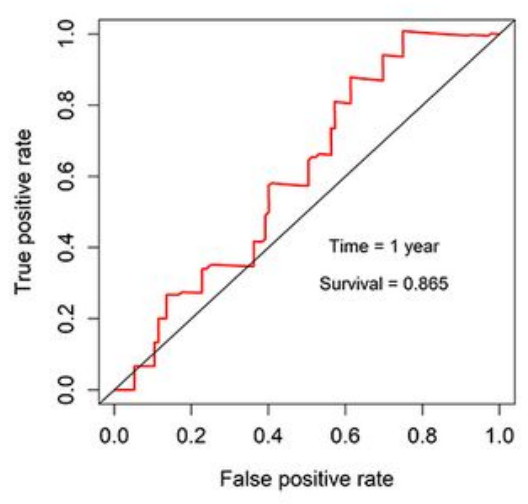

D

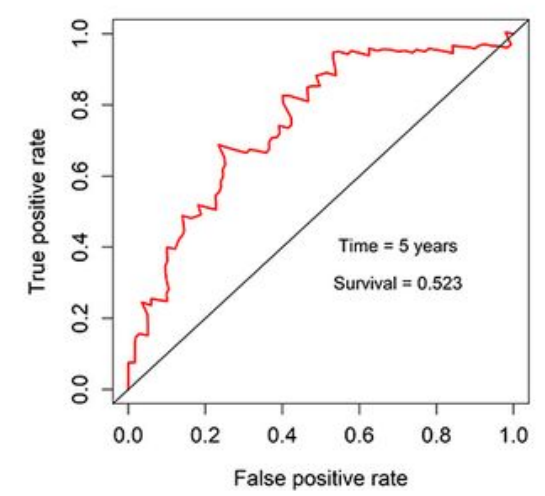

$\mathrm{F}$

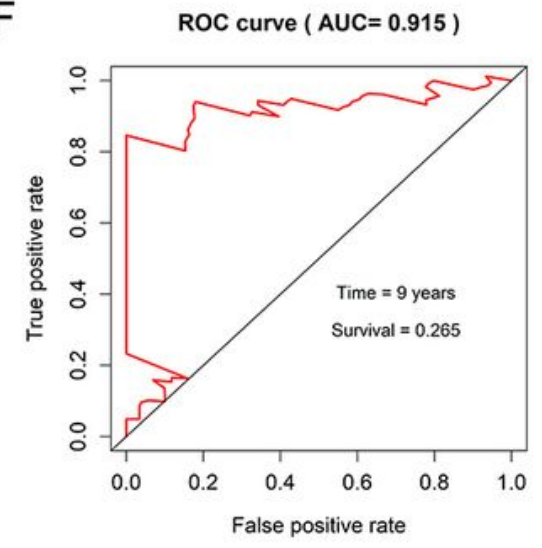

\section{Figure 7}

Examining the specificity and sensitivity of the prognosis model. (A) Kaplan-Meier survival curves. 111 LSCC patients were divided into two groups (high risk and low risk) according to the median of the risk score. (B-F) ROC curves. 

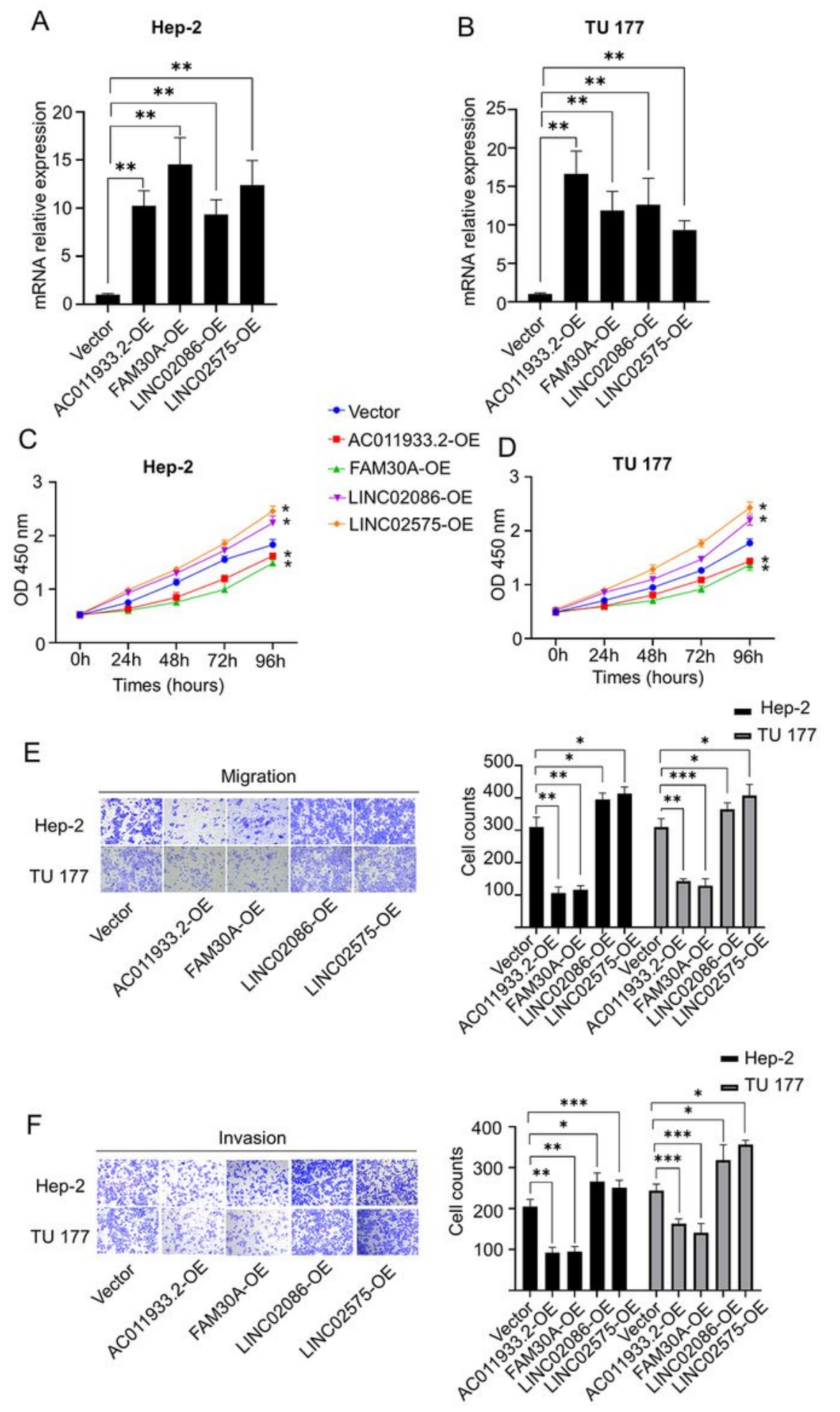

\section{Figure 8}

The effection of prognosis-related IncRNAs on LSCC cells. (A, B) We examined mRNA level by qPCR after Hep-2 and TU 177 were transfected plasmid containing prognosis-related IncRNA fragment. Vector represents the control group. AC011933.2-OE, FAM30-OE, LINC02086-OE, and LINC02575-OE represent overexpression groups of AC011933.2, FAM30, LINC02086, and LINC02575, respectively. (C, D) We detected cell viability at five-time points (0h, 24h, 48h, 72h, 96h) respectively after Hep-2 and TU 177 were 
transfected. (E, F) LSCC cells (Hep-2, TU 177) migration and invasion abilities were determined 48h after transfection. * indicating $p<0.05$. ** indicating $p<0.01$. *** indicating $p<0.001$.

\section{Supplementary Files}

This is a list of supplementary files associated with this preprint. Click to download.

- SupplementaryMaterial1.xlsx

- SupplementaryMaterial2.xlsx

- SupplementaryMaterial3.xlsx

- SupplementaryMaterial4.xIsx

- supplementarydata.docx 